\title{
Prevalence of Congenital Anomalies of Uterus in Sohag Government: A Descriptive Study by Trans-Vaginal Three Dimensional Ultrasound
}

\author{
SAYED A. SAYED, M.D.*; SALWA M. OUIES, M.D.** and BOTHYNA Z. ELSAYED, M.Sc.** \\ The Department of Human Anatomy and Embryology, Faculty of Medicine, Assuit* and Sohag** Universities
}

\begin{abstract}
Background: A large number of uterine anomalies are detected routinely in reproductive medicine. The prevalence of these congenital uterine anomalies in women with reproductive failure remains unclear especially in Upper Egypt.

The Aim of Study: Was to estimate the prevalence of congenital uterine anomalies in women with infertility in Sohag by Transvaginal Three Dimensional Ultrasound (TV3DUS).

Methods: This study was a descriptive cross-sectional study carried out for 977 infertile women in 2018 based on reviewing of radiological images at Sohag governorate. After taking informed and written consent, a detailed history, general and systemic examination was done. All the cases underwent TV3DUS performed during the postmenstrual phase. Examinations had been performed using flips iu 220 machine by a single operator and was commented as either normal or abnormal uterine cavity.
\end{abstract}

Results: A total of 977 women were included in the study. $808(82.7 \%)$ had a normal uterus and $169(17.3 \%)$ had a congenital uterine anomalies. The most common anomalies were arcuate $[n=84(49.7 \%)]$ and septate $[n=62(36.8 \%)]$ uterus, followed by unicornate $[\mathrm{n}=9(5.3 \%)]$ and then $\mathrm{T}$-shaped and hypoplastic uterus $[\mathrm{n}=4$ each $(2.3 \%$ each)], lastly didelphys and bicornate $[\mathrm{n}=3$ each $(1.8 \%$ each $)]$.

Conclusions: TV3D ultrasound is an accurate and simple method to detect and classify congenital uterine anomalies and should become the first diagnostic step in the assessment of the uterine cavity. Prevalence of uterine congenital anomalies in Sohag was $17.3 \%$. The most common type of uterine congenital anomalies was arcuate uterus.

Key Words: Arcuate - Infertility - Ultrasound - Uterus Anomalies

\section{Introduction}

THE organogenesis, morphogenesis and the differentiation of the female urogenital system are

Correspondence to: Dr. Sayed A. Sayed, The Department of Human Anatomy and Embryology, Faculty of Medicine, Assuit University amultifactorial and complex process. The uterus develops from paired Müllerian tubes. Congenital anomalies are the result of absence of fusion or reabsorption of the septum in-between the tubes or agenesis of one or two of them [1].

American Fertility Society classify Uterine anomalies into seven groups (1- Hypoplasia/aplasia. 2- Unicornate uterus. 3- Didelphic uterus. 4Bicornate uterus. 5- Septate uterus. 6- Arcuate uterus. 7- Diethylstilbestrol (DES) drug related uterus [2].

Another classification was based on 47 studies from 14 countries according to the distribution of these anomalies. The distribution of uterine anomalies is hypoplastic, aplastic, or soild (4\%); septate uterus (34\%); bicornuate (39\%); arcuate uterus (7\%); didelphic uterus (1 1\%); and unicornute uterus (5\%) [3]

Most uterine anomalies result from an error in the formation or fusion of the Mullerian ducts during embryogenesis [4]. Arcuate uterus is the commonest uterine anomaly and is only a mild deviation from the normally developed uterus, uterus appears with a smooth external contour and an internal indentation and it is the mildest degree of un-fused Mullerian ducts. Septate uterus results from failure of resorption of the partition between the two fused Müllerian ducts. The partition between the ducts is thought to resorb in a caudal to cranial direction. Failure of complete resorption results in a fibromuscular septum that can be partial or complete-dividing the uterine cavity and cervical canal into two parts. Complete failure of fusion of the paired Mullerian ducts results in duplication of the uterine corpus and cervix called uterus didelphys [5] . A bicornuate uterus results from failure of the Mullerian ducts to completely fuse, the central myometrium may extend to the level 
of the internal cervical os (bicornuate-unicollis) or external cervical os (bicornuate-bicollis) [6] Failure of one of the Mullerian ducts to completely or partially develop results in a unicornuate uterus. There are four types of unicornuate uteri: Unicornuate uterus with a communicating rudimentary horn, with a non communicating rudimentary horn, with or without a cavity and an isolated unicornuate uterus. Further, approximately $40 \%$ of patients with a unicornuate uterus will have an associated urinary tract anomaly [7].

Several techniques are available for the evaluation of Mullerian duct anomaly, among which the traditional Hysterosalpingography (HSG) and an invasive method which combines hysteroscopy and laparoscopy has been suggested as the gold standard for achieving a final diagnosis. Recently, noninvasive methods such as three-dimensional (3-D) ultrasound has been added, with their ability to demonstrate uterine contours, and therefore to improve the detection and differentiation between mullerian duct anomalies $[\mathbf{8 , 9}]$.

The aim of this study was to detect the prevalence of the congenital anomalies of the uterus in infertile women by 3D US in Sohag governorate.

\section{Patients and Methods}

This study was carried out for 977 infertile women in Sohag.

After taking informed and written consent, a detailed history, general and systemic examination was done. All the cases underwent TV3D ultrasound (fixed zoom) which was performed during postmenstrual phase. Examinations had been performed using flips iu220 machine by a single operator (at Sohag Materno-fetal center) in 2018 and was commented upon as either normal or abnormal uterine cavity.

\section{Inclusion criteria:}

All married females between 18 to 45 years complained from primary or secondary infertility was included.

\section{Exclusion criteria:}

1- Other causes of infertility.

2- Age less than 18 years and more than 45 years.

\section{Results}

The study based on reviewing of radiological images of the uterus by 3DUS at Sohag governorate. 977 infertile women were included.

\section{A- Prevalence of congenital anomalies:}

It was noticed that $(82.7 \% \mathrm{n}=808)$ females in the current study had normal 3D US findings and $(17.3 \% \mathrm{n}=169)$ had abnormal findings as shown in Graph (1), so the prevalence of uterine congenital anomalies among infertile women in Sohag governorate was $17.3 \%$.

Normal shaped uterus: By 3D US uterus the uterus appeared with a smooth external contour and the endometrium appeared triangular in shape. Fig. (1).

B- Types of congenital anomalies:

1-Arcuate uterus: The uterus appeared with a smooth external contour and internal indentation $\geq 1 \mathrm{~cm}$ but $<1.5 \mathrm{~cm}$ as shown in Fig. (2). It represented the most common congenital anomaly in the present study. There were 84 cases of 977 of infertile woman, which represented about $49.7 \%$ of congenital uterine anomalies in the present study.

2- Septate uterus: The uterus appeared with a flat external contour with an acute angle between the 2 endometrial cavities which are closely separated by septum may be partial or complete. It represented the second common congenital anomaly in the present study. There were 62 cases of 977 of infertile woman, which represented $36.8 \%$ of congenital uterine anomalies in the present study Fig. (3).

3-Bicornate uterus: The uterus appeared with an indentation at the external surface and with two endometrial cavities which were widely separated. There were 3 cases of 977 of infertile woman, which represented $1.8 \%$ of congenital uterine anomalies in the present study Fig. (4).

4- Unicornate uterus: The endometrial cavity assumed a fusiform (banana type) shape by 3DUS. The uterus usually shifted off the midline. There were 9 cases of 977 of infertile woman, which represented $5.3 \%$ of congenital uterine anomalies in the present study Fig. (5).

5- Didelphys uterus: There were complete duplication of the uterine corpus and cervix as seen by 3DUS. There were 3 cases of 977 of infertile woman, which represented $1.8 \%$ of congenital uterine anomalies in the present study Fig. (6).

6- T shaped uterus: T-shaped uterus referred to a specific radiographic appearance of the endometrial cavity as a T-shape as seen by 3DUS. There were 4 cases of 977 of infertile woman, which represented about $2.3 \%$ of congenital uterine anomalies in the present study Fig. (7). 
7- Hypoplastic uterus: The uterus was normally appeared, but with a small size as seen by 3DUS. There were 4 cases of 977 of infertile woman, which represented $2.3 \%$ of congenital uterine anomalies in the present study Fig. (8).

The most common congenital anomalies was arcuate uterus, presented with 84 cases $(49.7 \%)$ of the abnormal finding, then septate uterus presented with 62 cases $(36.8 \%)$ followed by unicornate uterus 9 cases $(5.3 \%)$ and then T-shaped and hypoplastic uteri 4 cases each $(2.3 \%$ each $)$, lastly didelphys and bicornate uterus 3 cases ( $1.8 \%$ each) Gragh (2).

\section{C- Prevalence of infertility among the studied group:}

In the present study about $(65 \% \mathrm{n}=635)$ females had primary infertility and $(35 \% \mathrm{n}=342)$ had secondary infertility. As regard the association between uterine anomalies and infertility it was found that $68 \%$ of females with uterine congenital anomalies presented with 1ry infertility and $32 \%$ presented with 2ry infertility Graph (3).

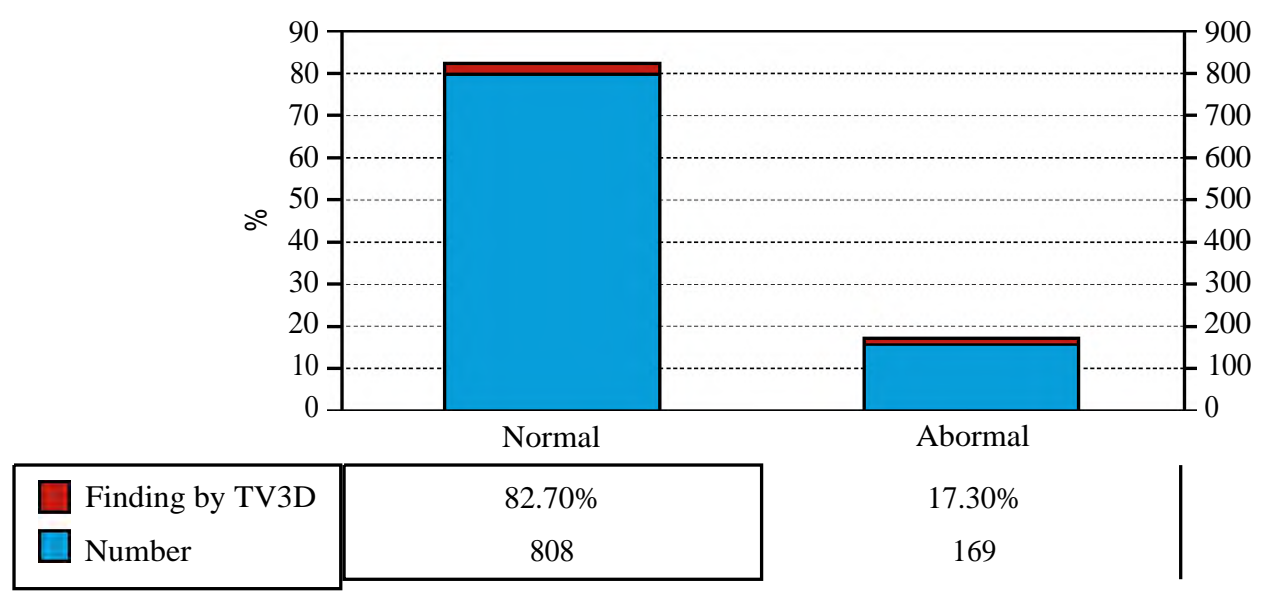

Graph (1): Prevalence of congenital anomalies in 977 infertile women.

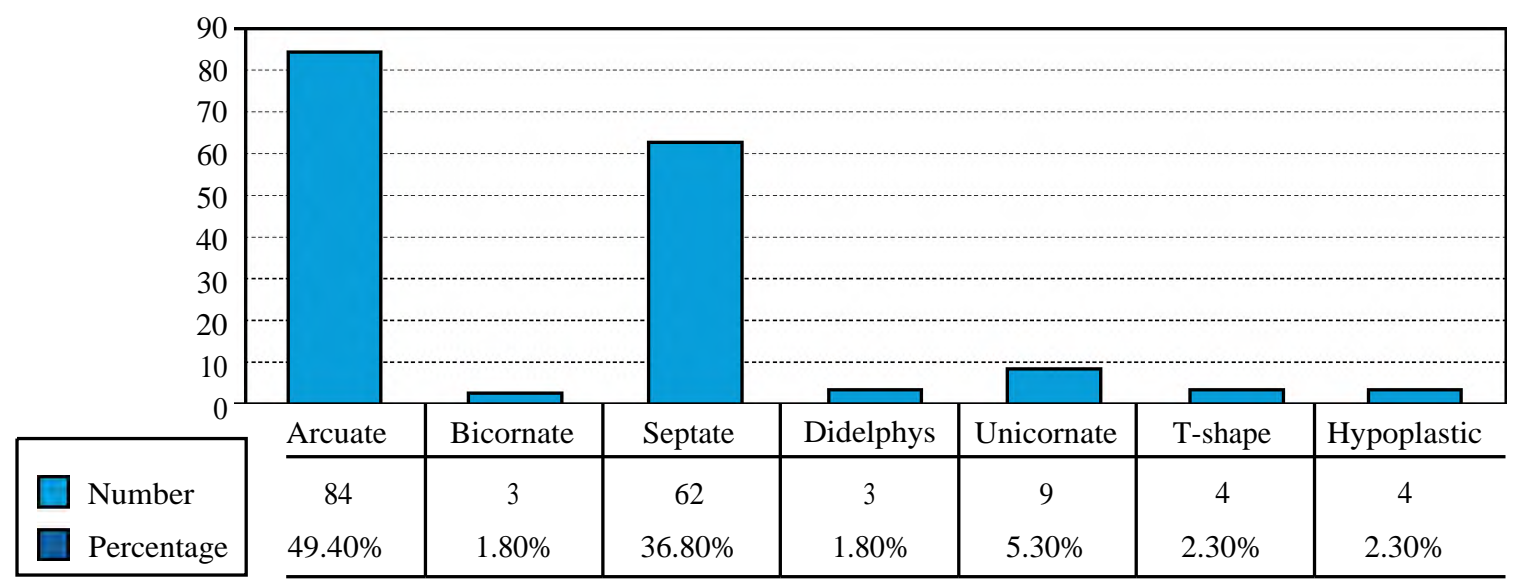

Graph (2): Types of congenital anomalies in 977 infertile women.

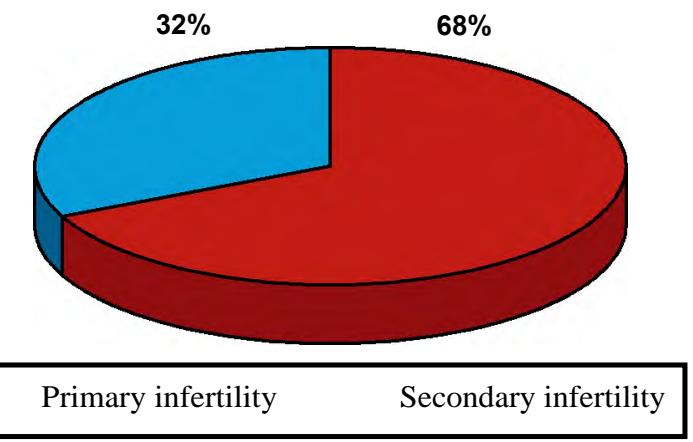

Graph (3): Prevalence of infertility in relation to uterine congenital anomalies (169 cases).

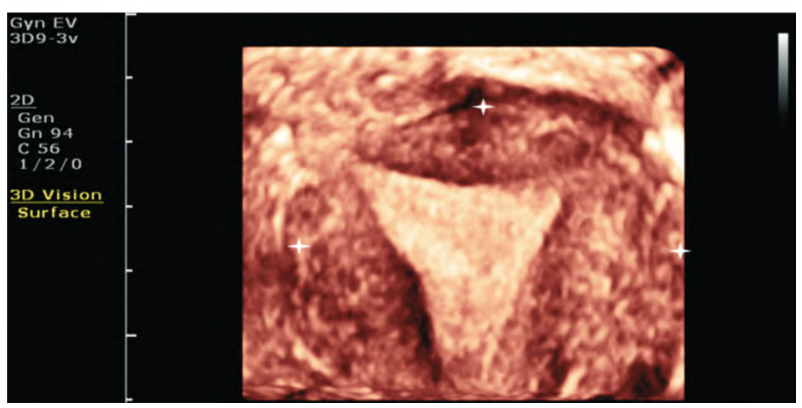

Fig. (1): 3D ultrasound image of the normal appearance of the uterus with a smooth external contour (stars). The endometrium is triangular in shape. 


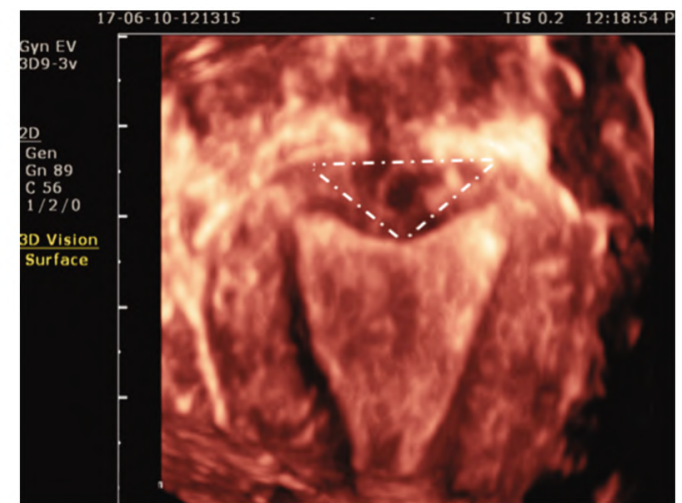

Fig. (2): 3D ultrasound image of an arcuate uterus with a smooth external contour and internal indentation $\geq 1 \mathrm{~cm}$ but $\leq 1.5 \mathrm{~cm}$ (dotted lines).
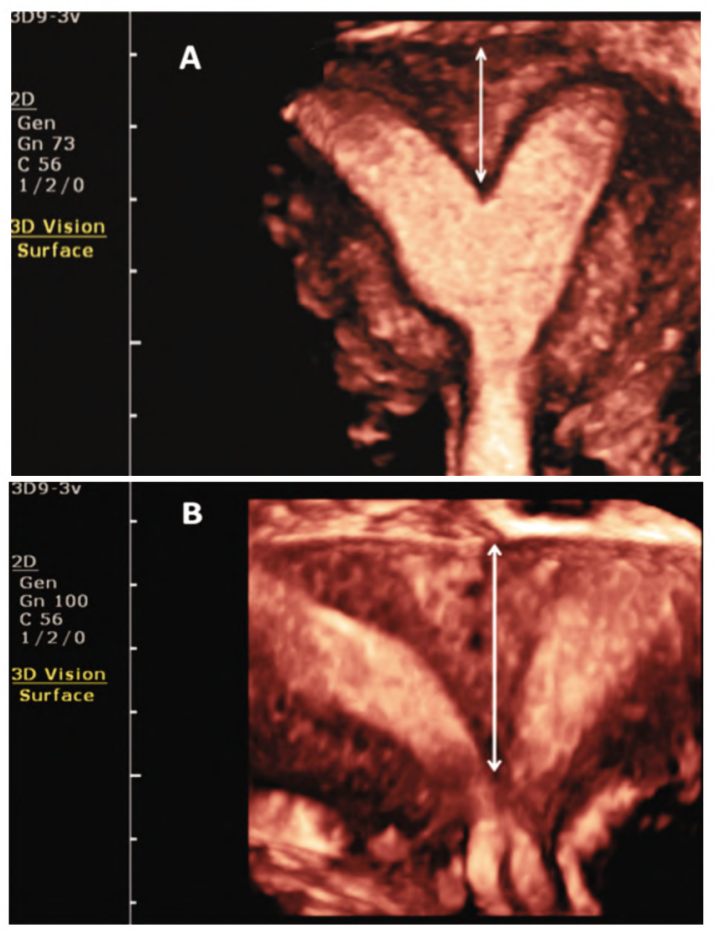

Fig. (3): 3D ultrasound images of a septate uteri (A, B) There is flat external contour, acute angle between the endometrial cavities which are closely separated by septum may be partial (arrow A) or complete (arrow B).

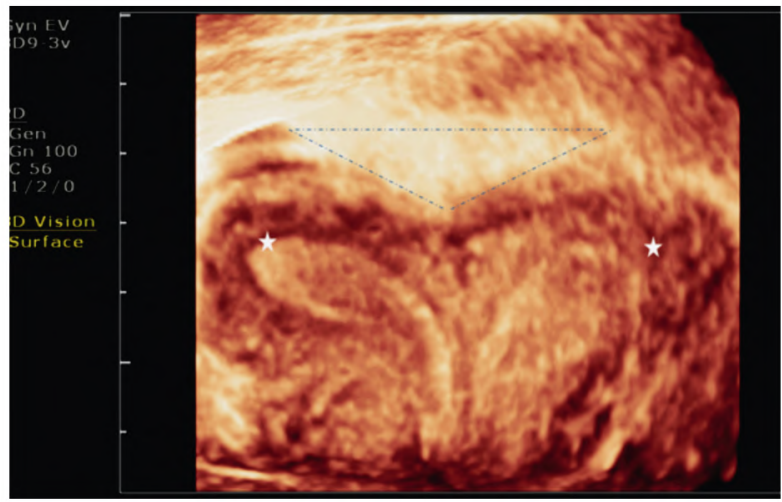

Fig. (4): 3D ultrasound image of bicornate uterus ( 2 corns $=$ stars), there is indentation at the external surface (dotted lines) between the two endometrial cavities which are widely separated.

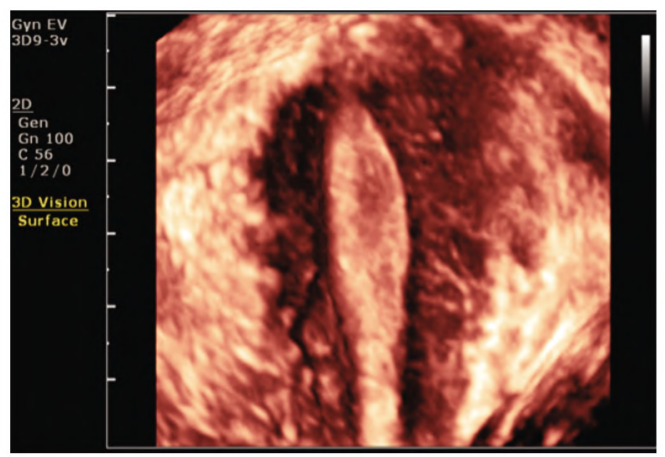

Fig. (5): 3D ultrasound image of unicornate uterus, the endometrial cavity assumes a fusiform (banana type) shape. The uterus shifted off the midline.

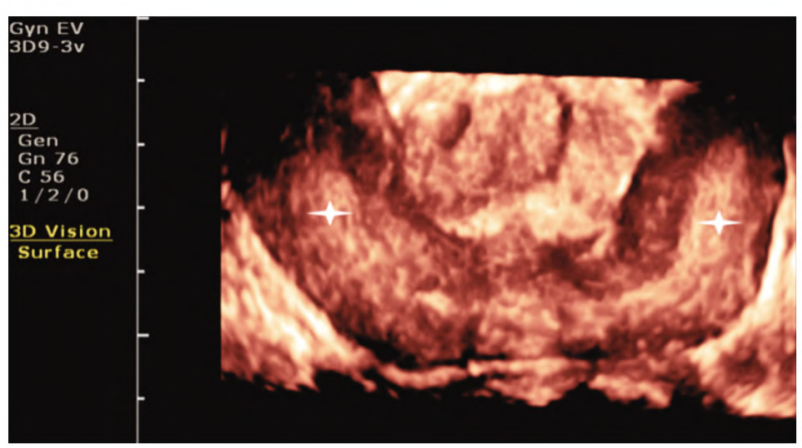

Fig. (6): 3D ultrasound image of Didelphys uterus, there is complete un-fusion between the cervix and the hemi uterine cavity. In each uterus (star), normal uterine zonal anatomy is preserved.

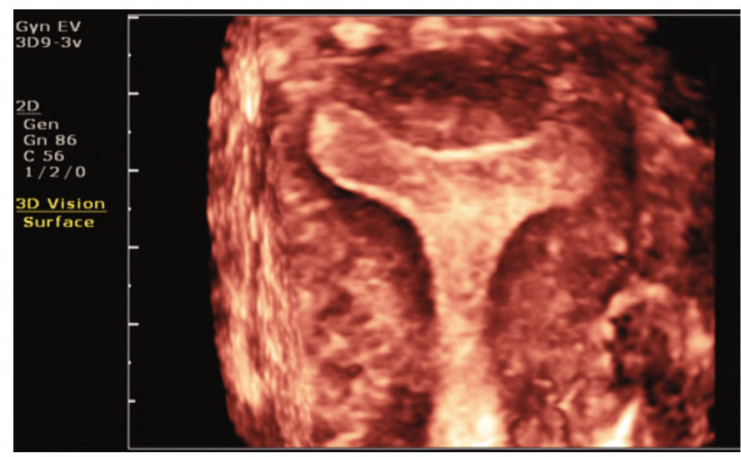

Fig. (7): 3D ultrasound images of T-shaped uterus. The endometrial cavity is T-shaped.

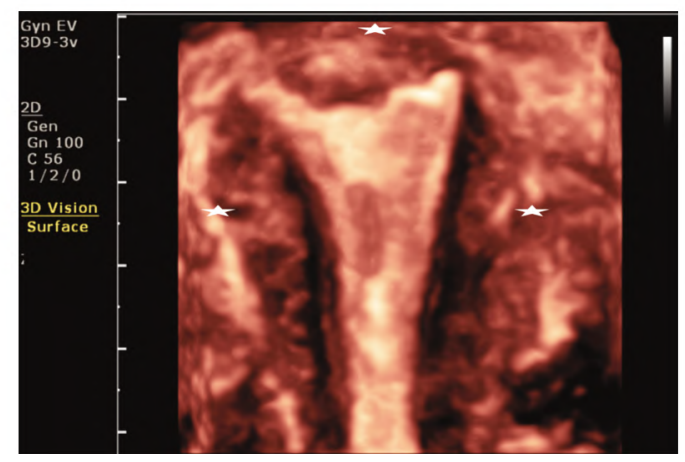

Fig. (8): 3D ultrasound images of hypoplastic uterus (appear as normal uterus but with small size). The uterus appears with smooth external contour (stars), triangular cavity and a thin muscular wall. 


\section{Discussion}

In general, TVUS as a noninvasive and valuable diagnostic modality plays an important role in the evaluation of uterus and endometrial abnormalities [10].

The prevalence of uterine congenital anomalies among infertile women in Sohag governorate was $17.3 \%$. The most common congenital anomalies in this study was arcuate uterus present $49.7 \%$ of abnormal finding, septate uterus (36.8\%), unicornate uterus (5.3\%), T-shaped and hypoplastic uterus (2.3\% each), lastly didelphys and bicornate uterus (1.8\% each).

In a previous study using 1976 case in united kingdom by 3DUS the prevalence of congenital anomalies was $5.3 \%$ in infertile women. The most common types of anomalies was arcuate uterus (3.6\%), sepatate uterus (1.4\%) and bicornate uterus $(0.2 \%)[11]$

In another study [12] using 1089 case in united kingdom using 3DUS, the prevalence of congenital anomalies was $9.4 \%$ in infertile women, the most common type was arcuate uterus $(6.6 \%)$, sepatate $(2.7 \%)$ and bicornate uterus $(0.5 \%)$.

A previous systemic review [13] showed that the prevalence of congenital uterine anomalies in unselected and high-risk populations was $5.5 \%$ in infertile women. Arcuate uterus was most common type in the unselected population.

In Croatia, Radoncic and Funduk in a study using 267 case found that the prevalence of congenital uterine anomalies was $36 \%$. The most common type was septate uterus $(35.6 \%)$ then arcuate uterus $(0.4 \%)$. No other types was detected [14].

Another study done by HSG/TVS in Iran using 186 case to detect uterine anomalies, the prevalence of congenital anomalies was $20 \%$ in infertile women, the most common type was arcuate uterus then subsepatae and unicornate uterus [15]

Apart from study done by Radoncic and Funduk in which septate uterus was the commonest, all the previous studies found that the most common anomaly was arcuate uterus.

In the present study about $68 \%$ of uterine congenital anomalies females presented with 1ry infertility and $32 \%$ presented with 2ry infertility.

Studies from 1950 to 2007 were identified through a MEDLINE search [16] suggested that the prevalence of congenital uterine anomalies was near $6.7 \%$ in the general population and about $7.3 \%$ in the infertile population.

In a study included 100 cases of primary infertile females over a period of 3 years from 2007 to 2009 in India, bicornuate uterus appeared in 4 cases out of the total cases. Septate uterus and uterus didelphys were observed in 2 cases each, whereas arcuate uterus and unicornuate uterus were observed in 1 case each with prevalence of $10 \%$ uterine anomalies in the study [17].

The cause of congenital uterine anomalies not well understood yet,some studies suggest the relation between T-shaped uterus and receiving oral contraceptive pills during pregnancy. T-shaped is associated also with failed implantation, increased risk of ectopic pregnancy, miscarriage and preterm delivery, which always needs a surgical procedure to correct the malformation [18]

\section{Conclusion:}

- TV3D ultrasound is an accurate and simple method to detect and classify congenital uterine anomalies and should become the first diagnostic step in the assessment of the uterine cavity.

- Prevalence of uterine congenital anomalies in sohag governorate was $17.3 \%$.

- Most common type of uterine congenital anomalies was arcuate uterus.

- Uterine congenital anomalies is associated always with primary infertility.

\section{Recommendations:}

- Furthermore study is needed to discover relation between unicornate uterus and urogenital ridge anomalies.

- Furthermore studies with more population is needed.

\section{References}

1- SPENCER T.E., DUNLAP K.A. and FILANT J.: Comparative developmental biology of the uterus: Insights into mechanisms and developmental disruption. Mol. Cell Endocrinol., 354: 34-53, 2012.

2- PAVONE M.E., KING J.A. and VLAHOS N.: Septate uterus with cervical duplication and a longitudinal vaginal septum: A müllerian anomaly without a classification. Fertil Steril., 85: 494. e9-494. e10, 2006.

3- NAHUM G.G.: Uterine anomalies. How common are they, and what they distribution among subtypes? Rep. Med., 43; 10: 877: 87, 1998.

4- TROIANO R.N. and McCARTHY S.M.: The Pelvis University College Cork Archived from the original on 2008- 
02-27 Müllerian Duct Anomalies: Imaging and Clinical Issues. Radiology, 233: 19-34, 2004.

5- ACIEN P.: Incidence of Mullerian detects in fertile and infertile women. Hum Reprod, 12: 1372-6, 1997.

6- GRIMBIZIS G.F., CAMUS M., TARLATZIS B.C., BONTIS J.N. and DEVROEY P.: Clinical implications of uterine malformations and hysteroscopic treatment results. Hum. Reprod Update, 7: 161-74, 2001.

7- FEDELE L., BIANCHI S., AGNOLI B., TOZZI L. and VIGNALI M.: Urinary tract anomalies associated with unicornuate uterus. J. Urol., 155: 847, 1999.

8- REUTE K.L., DALY D.C. and COHEN S.M.: Septate versus bicornuate uteri: Errors in imaging diagnosis. Radiology, 172: 749-52, 1989.

9- STEINER H., STAUDACH A., SPITZER D. and SCHAFFER H.: Three-dimensional ultrasound in obstetrics and gynecology: Technique, possibilities and limitations. Human Reproduction, 9: p. 1773-8, 1994.

10-PRETORIUS D.H. and NELSON T.R.: Three-dimensional ultra sound imaging inpatient diagnosis and management: The future. Ultrasound Obstet. Gynecol., 1: 381, 1995.

11- SALIM R., WOELFER B., BACKOS M., REGAN L. and Jurkovic D.: Reproducibility of three-dimensional ultrasound diagnosis of congenital uterine anomalies. Ultrasound Obstet. Gynecol., 21: p. 578-82, 2003.

12- WOELFER B., SALIM R., BANERJEE S., ELSON J.,
REGAN L. and JURKOVIC D.: Reproductive outcomes in women with congenital uterine anomalies detected by three-dimensional ultrasound screening. Obstet. Gynecol., 98: 1099-103, 2001.

13- JAYAPRAKASAN C.K., ZAMORA J., THORNTON J.G. and RAINE-FENNING N.: The prevalence of congenital uterine anomalies in unselected and high-risk populations: A systematic review, Hum Reprod Update. Nov.; 17 (6): 761-71, 2011.

14- RADONCIC E. and FUNDUK-KURJAK B.: Threedimensional ultrasound for routine check-up in vitro fertilization patients. Croat. Med. J., 41; 262, 2000.

15- ALBORZI S., DEHBASHI S. and KHODAEE R.: Sonohysterosalpingographic screening forinfertile patients. Int. J. Gynaecol. Obstet., 82: 57-62, 2003.

16- SARAVELOS S.H., COCKSEDGE K.A. and LI T.C.: Prevalence and diagnosis of congenital uterine anomalies in women with reproductive failure: A critical appraisal. Hum. Reprod, 14; 5 p. 415-29, 2008.

17- JAYASHREE A., UDAYA KUMAR P., PADMAJA V., VINODINI L. and SUDHA RANI K.: An analysis of the role of uterine malformations in primary infertility-an observational study. Int. J. Cur. Res. Rev., 7; 16 p. 62-7, 2015.

18- MEIER, ROSE; CAMPO and RUDI: "T-Shaped Uterus". Female Genital Tract Congenital Malformations, 261-70, 2015.

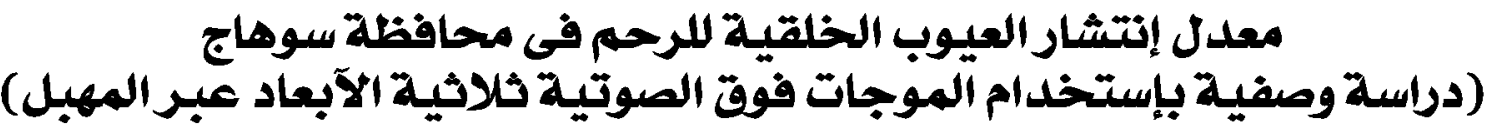

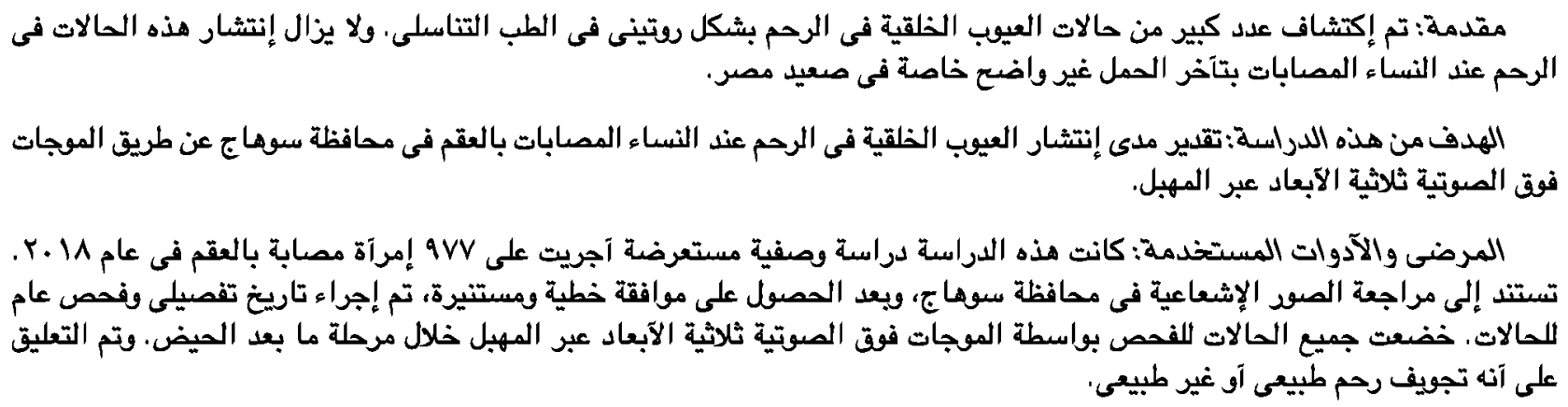

